Ann. Zootech., I977, 26 (2), I39-I48.

\title{
Variation de la stabilité du vide avec la capacité de la pompe et le volume intérieur d'une installation de traite mécanique
}

\author{
J. LE DU \\ avec la collaboration technique de P. LAMBIon \\ Laboratoive de Recherches sur la Traite, \\ Centre de Recherches de Revines, I.N.R.A., \\ 35042 Rennes Cedex (France)
}

\section{Résumé}

La stabilité du vide de traite a été étudiée sur une machine à traire expérimentale dont on fait varier le volume intérieur, V, de $80 \mathrm{l}$ à $280 \mathrm{l}$ et la capacité, C, de la pompe à vide, de $14^{\circ} \mathrm{Nl} / \mathrm{mn}$ à. $860 \mathrm{~N} 1 / \mathrm{mn}$.

Le test pratiqué consiste à introduire rapidement dans l'installation un volume d'air de Io Nl en branchant un pot à lait "standard" de 201 au niveau du lactoduc (fig. I). La perturbation produite est enregistrée par des capteurs de pression placés près du lactoduc et sur le petit tuyau à lait (fig. 2).

Les résultats présentés à la figure 3 et aux tableaux I et 2 montrent principalement que :

- l'accroissement de la capacité $C$ se traduit par une diminution de la durée des perturbations $(\mathrm{P}<\mathrm{O}, \mathrm{oI})$. Cet effet est d'autant plus accentué que $\mathrm{C}$ est faible. Globalement, la capacité de la pompe a peu d'incidence sur l'amplitude des perturbations lorsque le volume $V$ est supérieur ou égal à $x_{5}$ l.

- l'accroissement du volume $\mathrm{V}$ entraine une diminution marquée de l'amplitude des ébranlements $(\mathrm{P}<0,01)$ et une augmentation $(\mathrm{P}<0,0 \mathrm{I})$ faible de la durée.

- le produit $p=$ amplitude $\times$ durée, choisi pour quantifier la stabilité du vide, décroît lorsque $\mathrm{C}$ et $\mathrm{V}$ augmentent. Cette décroissance est toutefois d'autant moins accentuée que ces deux valeurs tendent vers les valeurs les plus élevées. Il en résulte qu'au-delà d'un certain seuil, l'amélioration de la stabilité du vide que l'on obtient en augmentant la puissance de la pompe et le volume intérieur d'une installation devient imperceptible. L'existence de ce seuil semblerait en partie due au fonctionnement du régulateur de vide et aux pertes de charges du circuit.

- en ce qui concerne le contrôle à la ferme, le produit de l'amplitude par la durée de l'ébranlement semble par conséquent le mieux approprié pour quantifier la stabilité du vide. Toutefois, ce critère ne mettra en évidence des différences entre installations qu'à condition que les volumes intérieurs et les puissances de pompes soient relativement faibles. 


\section{Introduction}

On distingue en général deux types de fluctuations du vide à l'intérieur du manchon durant la traite (NyHAN, Ig68) :

- Les fluctuations " cycliques ", de même fréquence que la pulsation sont associées au mouvement du manchon et à l'écoulement du lait.

- Les fluctuations irrégulières ou " acycliques " sont dues aux entrées d'air provoquées par la vidange de bocaux ou d'extracteurs, la pose, la dépose ou la chute de faisceaux trayeurs.

De nombreux auteurs, cités par NyHAN (1968) considèrent que l'instabilité du niveau de vide a une incidence nocive sur l'état sanitaire de la mamelle. Il est en outre fait état d'un accroissement des temps de traite (STANLEY, KEsLER, Borthree, I962) ainsi que d'une diminution du débit moyen et du débit maximum (Schmidt et al., ig64, Nyhan et Cowhig, I967).

Bien que l'on admette que l'accroissement du volume intérieur d'une installation ou de la capacité volumétrique de la pompe améliore la stabilité du vide de façon importante (PHILLIPS, I952; FELL, I963), les valeurs recommandées sont très diverses. Ainsi, pour I2 postes de traite, la capacité de pompe préconisée serait de $6351 / \mathrm{mn}\left({ }^{1}\right)$ en Irlande $\left({ }^{2}\right), 870 \mathrm{Nl} / \mathrm{mn}$ en France $\left({ }^{3}\right)$, I $680 \mathrm{Nl} / \mathrm{mn}$ aux États-Unis $\left({ }^{4}\right)$. De façon similaire, la capacité du récipient intercepteur, destiné à accroître le volume intérieur de l'installation, serait dans ce cas de 201 en France $\left({ }^{3}\right)$ et de 2281 en Californie ( $\left.{ }^{5}\right)$.

Or, les travaux récents semblent montrer qu'au-delà de certaines valeurs minimales, l'amélioration de la stabilité du vide obtenue en continuant à accroître les puissances et les volumes devient minime par rapport aux investissements à consentir. Ainsi, pour de grandes variations de capacité de pompe, Rossing (I970) et WOOLFORD (I974) ne constatent que de faibles variations de la stabilité du vide alors que les modifications du volume intérieur auraient dans certains cas, un effet prépondérant.

Dans ce contexte, nous présentons ci-après une étude des perturbations du niveau de vide enregistrées dans une machine à traire lors de l'entrée soudaine de faibles quantités d'air. Cette expérimentation, conduite en faisant varier la capacité de la pompe (I40 à $860 \mathrm{Nl} / \mathrm{mn}$ ) et le volume intérieur de l'installation ( 80 à 280 l), a pour objet de vérifier l'intérêt de la méthode proposée par Rossing (I973) pour le contrôle de la stabilité du vide à la ferme et de mieux comprendre les conditions d'établissement des pressions dans une installation de traite.

\section{Matériel et méthodes}

\section{A. - Installation expérimentale}

L'installation de traite expérimentale, construite dans notre laboratoire, est décrite à la figure I. La pompe à vide, dont la capacité volumétrique est ajus-

(r) N1 : litre d'air dans les conditions de l'atmosphère normale (température : $20{ }^{\circ} \mathrm{C}$; pression : $760 \mathrm{~mm} \mathrm{Hg}$ ).

(2) Milking : Equipment and installations (1973). An Foras Taluntais, 33 Merrion Road - Dublin 4.

(3) Projet de norme expérimentale U 55 _... : Installations de traite mécanique : Règles de construction et performances. Comité Français de la Traite Mécanique. I49, rue de Bercy, 750r 2 Paris.

(4) $3 \mathrm{~A}$ accepted practices for the design, fabrication and installation of milking and milk handling equipment (I968). J. Milk. Fd. Technol. 3I (I I) 355-365.

(5) Publ. Univ. Calif. Agric. Ext. Service A X T - 94 (I964) cité par MerN G. A. (I968) in Components and standardization. Proc. Symp. Machine Milking-Reading. 27-4I. 


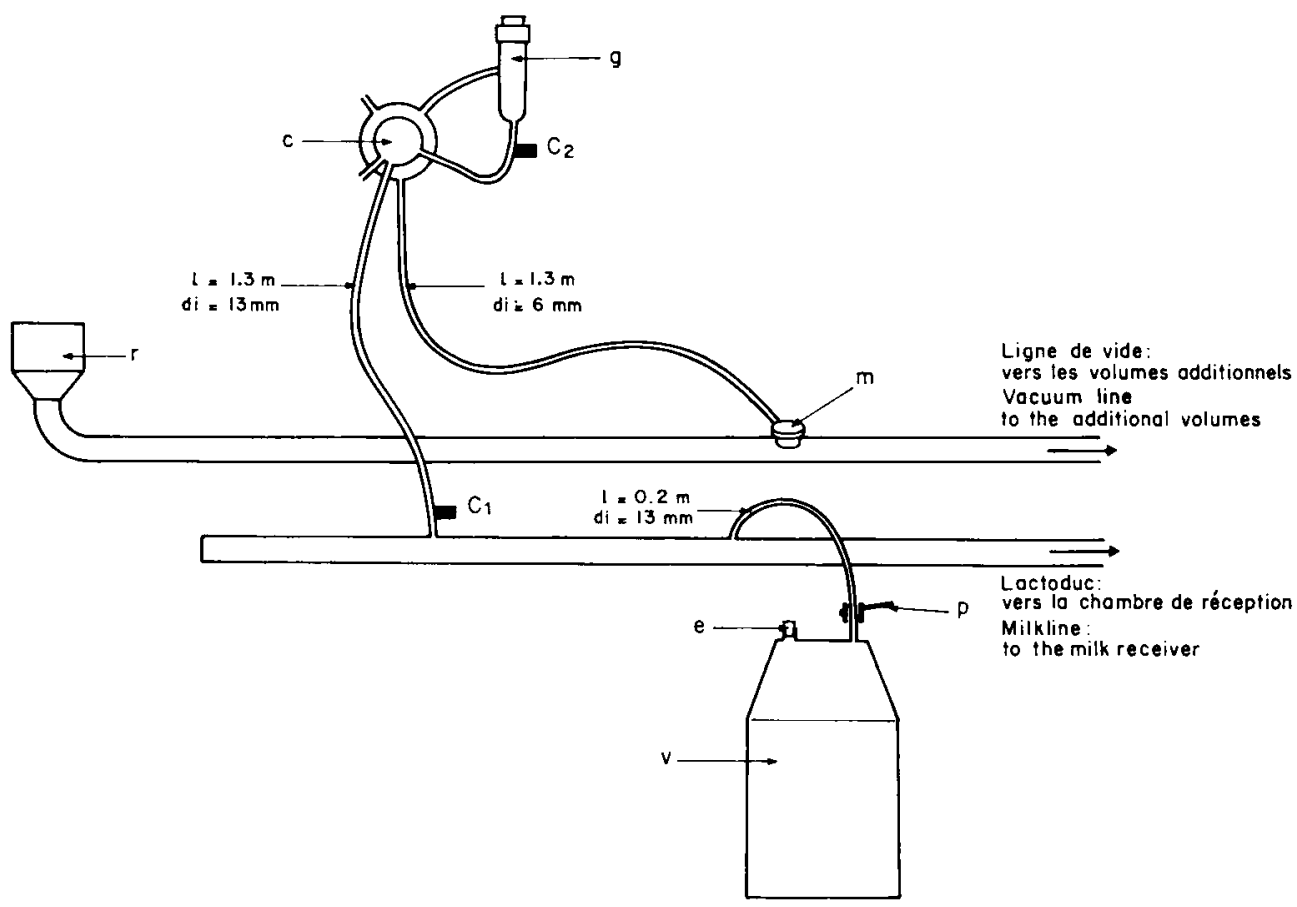

FIG. 1. - Dispositif expérimental:

$g: 4$ gobelets dont les manchons sont bouchés; $c:$ griffe (entrée d'air; $d i=0,7 \mathrm{~mm}$ et volume I 70 ml); $r$ : régulateur à poids; $m$ : relais de pulsateur; $v$ : pot à lait d'un volume de 201 ; $e:$ bouchon pour remise du pot $v$ sous pression atmosphérique; $p$ : pince pour l'admission d'air.

\section{Experimental equipment:}

$g: 4$ teat cups with stoppered liners; $c:$ claw (air admission; $D i=0,7 \mathrm{~mm}$ and volume : $\mathbf{1} 70 \mathrm{ml}$ ); $r:$ weighted regulator; $m:$ pulsator relay; $v:$ bucket of 201 volume; $e:$ stopper for admission ef air at atmospheric pressure in bucket $v ; p$ : clip for air admission.

table entre Ioo $\mathrm{Nl}$ et I $400 \mathrm{Nl} / \mathrm{mn}$, est reliée à une canalisation de vide $(l=8,3 \mathrm{~m}$ di $=42 \mathrm{mn})$ ainsi qu'à un lactoduc $(l=4,0 \mathrm{~m}$; di $=40 \mathrm{mn})$. Les autres composants : un récipient intercepteur $(23 \mathrm{l})$, une chambre de 1éception (24 1 ), un piège sanitaire $(6 \mathrm{l})$ et un faisceau trayeur portent le volume intérieur Vo de l'installation à 801 . Ce volume initial peut être accru en interposant sur le circuit de vide, à $3 \mathrm{~m}$ de la pompe, un récipient intercepteur additionnel de capacité variable : 40, 70 , IOO, I40 et $200 \mathrm{l}$. Un relais de pulsateur (Rapport $75 \%$; Vitesse : $60 \% / \mathrm{mn}$ ) est monté à $\mathrm{I}, 5 \mathrm{~m}$ du régulateur $(50 \mathrm{kPa})\left({ }^{1}\right)$. Le faisceau trayeur et le pot $(\mathrm{v})$ sont reliés au lactoduc respectivement à $0,4 \mathrm{~m}$ et $\mathrm{I}, 2 \mathrm{~m}$ de l'extrémité libre de celui-ci.

\section{B. - Méthode d'enregistrement}

La pompe et le système de pulsation étant en fonctionnement et la pince $(p)$ fermée (fig. I), le bouchon (e) est enlevé puis remis en place. L'ouverture de la pince $(p)$, en provoquant l'entrée de so Nl d'air dans le système, crée une perturbation

(I) Ioo kilo Pascal $(\mathrm{kPa})=750 \mathrm{~mm} \mathrm{Hg}$. 
du niveau de vide qui est analysée par le capteur $C_{2}\left({ }^{1}\right)$ sur le petit tuyau à lait et par le capteur $\mathrm{C}_{\mathbf{1}}{ }^{(1)}$ sur le long tuyau à lait, à $\mathrm{I}$ o $\mathrm{cm}$ du lactoduc. Les signaux sont recueillis par un enregistreur oscillographique à trace directe $\left(^{2}\right)$ aux échelles suivantes : 5o kilo Pascal pour $20 \mathrm{~cm}$ et une seconde pour 2 ou $4 \mathrm{~cm}$.

\section{C. - Paramètres étudiés}

L'ébranlement, qui affecte les formes présentées figure 2, est caractérisé par : la durée de la première oscillation $d$, en seconde, l'amplitude de la première oscillation $a$, en kilo Pascal et le produit $p=a \times d$, en kilo Pascal.seconde.

Il nous est apparu au cours d'essais préliminaires que la forme de l'ébranlement n'est pas sensiblement affectée par la distance de propagation dans les

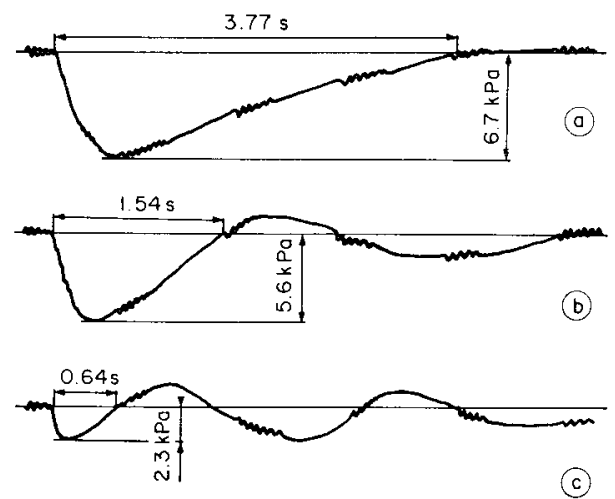

FIG. 2. - Allure des perturbations du niveau de vide enregistrées.

Chaque ébranlement est caractérisé par la durée (en secondes) et l'amplitude (kilo Pascal) de la première fluctuation (roo $k \mathrm{~Pa}=750 \mathrm{~mm} \mathrm{Hg}$ ).

Aspect of recorded vacuum fuctuations.

Every recording is characteristd by the duration (seconds) and amplitude of the first fluctuation ( (оо $k \mathrm{~Pa}=750 \mathrm{~mm} \mathrm{Hg}$ ).

canalisations (jusqu'à $6 \mathrm{~m}$ dans nos essais et avec interposition d'une chambre de réception sur le circuit). Il en résulte que les enregistrements donnés par les capteurs $\mathrm{C}_{1}$ et $\mathrm{C}_{2}$ sont pratiquement semblables. Toutefois, en $\mathrm{C}_{2}$, la pulsation transmet au petit tuyau à lait une vibration parasite qui rend plus difficile la détermination exacte de la durée et de l'amplitude de l'ébranlement. En conséquence, nous présentons, ci-après, pour chaque combinaison de capacité de pompe, $C$, et de volume intérieur, $\mathrm{V}$, les valeurs moyennes de 4 enregistrements effectués au niveau du lactoduc (Capteur $\mathrm{C}_{1}$ ).

(r) Marque Endevco. Type $8503 \pm 40$ p. s. i. Représenté par Endevco, 76, rue des Grands-Champs, 75020 Paris. Paris.

(2) Marque Southern instruments. Type I0.400. Représenté par Sedeme II, rue Simonet, 750 I3 


\section{Résultats}

\section{A. - Aspects des perturbalions}

Pour les plus faibles capacités de pompe, le système revient à sa position d'équilibre sans jamais la dépasser (fig. $2 a$ : mouvement apériodique) tandis qu'aux plus fortes capacités de pompe correspond une fluctuation périodique du niveau de vide (fig. $2 b$ ). Notons que la première oscillation peut être d'amplitude et de durée égales ou inférieures à celles des suivantes lorsqu'on est voisin de la capacité maximale du régulateur (fig. $2 c$ ).

\section{B. - Durée des perturbations: $\mathrm{d}$}

L'accroissement de la capacité volumétrique $C$, de la pompe se traduit par une réduction hautement significative de la durée $d$ de la perturbation lorsque la pompe est de faible puissance. Toutefois, l'effet s'annule pour les plus fortes capacités de pompe quel que soit $\mathrm{V}$ (fig. 3 et tabl. I).

\section{TABLEAU I}

Effet de l'accroissement de la capacité de la pompe (l/mn) sur la duvée, d, l'amplitude, a et le produit a $\times \mathrm{d}$, des ébranlements pour des volumes d'installation de 80, I8o et $280 l$

Effect of increasing vacuum pump capacity $(l / m n)$ on the duration $\mathrm{d}$, the amplitude a, and the product a $\times \mathrm{d}$, of the vacuum fuctuations, for internal plant volumes of 80,180 and $280 l$

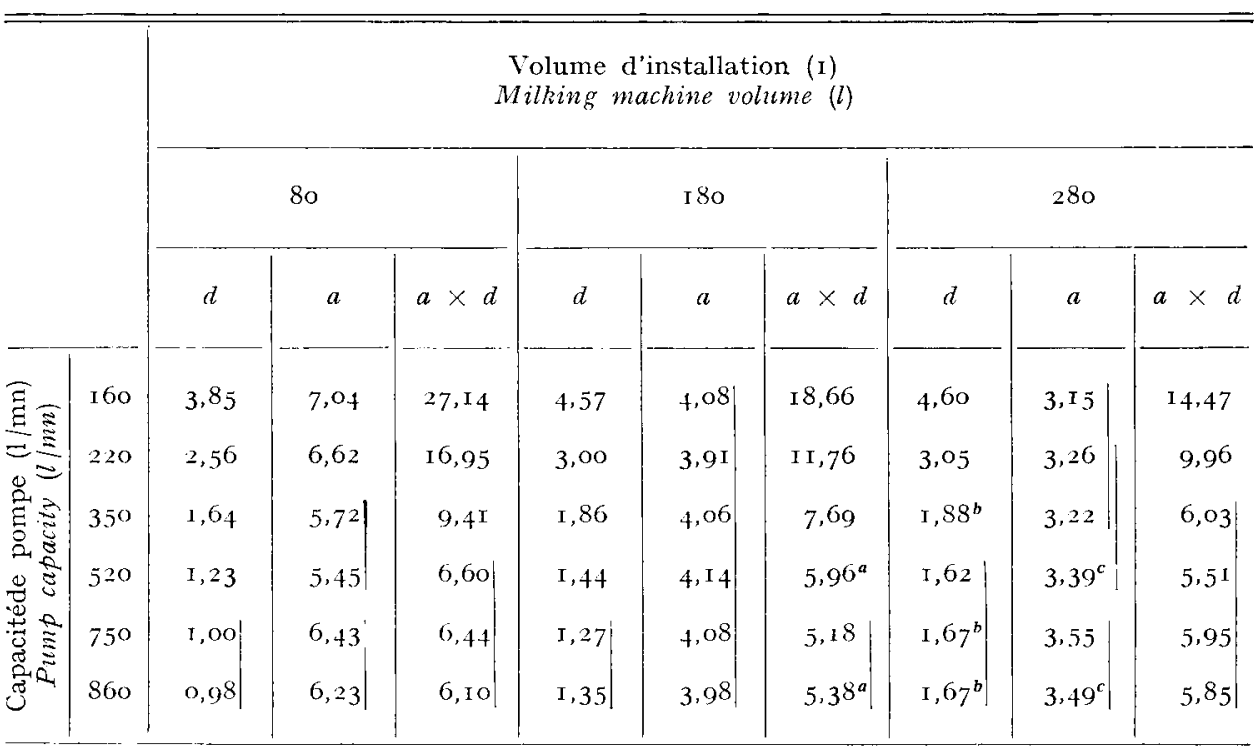

Les valeurs réunies par un même trait vertical ou repérées par une même lettre ne diffèrent pas entre elles au niveau de probabilité de $5 \mathrm{p}$. I 00 . Du fait de l’hétérogénéité des variances, des tests " $t$ » ont été effectués.

The values gathered by the same line or sharing a common letter are not significantly different $(\mathrm{P}<0.05)$.

Because of variance heterogeneity, the values were compared by means of the " $\mathrm{t}$ "test. 
L'augmentation du volume intérieur $\mathrm{V}$ de l'installation n'entraîne qu'un faible allongement de la durée d'un ébranlement $(3,85 \mathrm{~s}$ et $4,60 \mathrm{~s}$ pour $\mathrm{C}=\mathrm{I} 6 \mathrm{o} \mathrm{Nl}$ / $\mathrm{mn} ; 0,98 \mathrm{~s}$ et $\mathrm{I}, 67 \mathrm{~s}$ pour $\mathrm{C}=860 \mathrm{Nl} / \mathrm{mn}$ lorsque $\mathrm{V}$ croît de $80 \mathrm{l}$ à $280 \mathrm{l})(\mathrm{P}<0,0 \mathrm{r})$. (fig. 3 et tabl. 2).
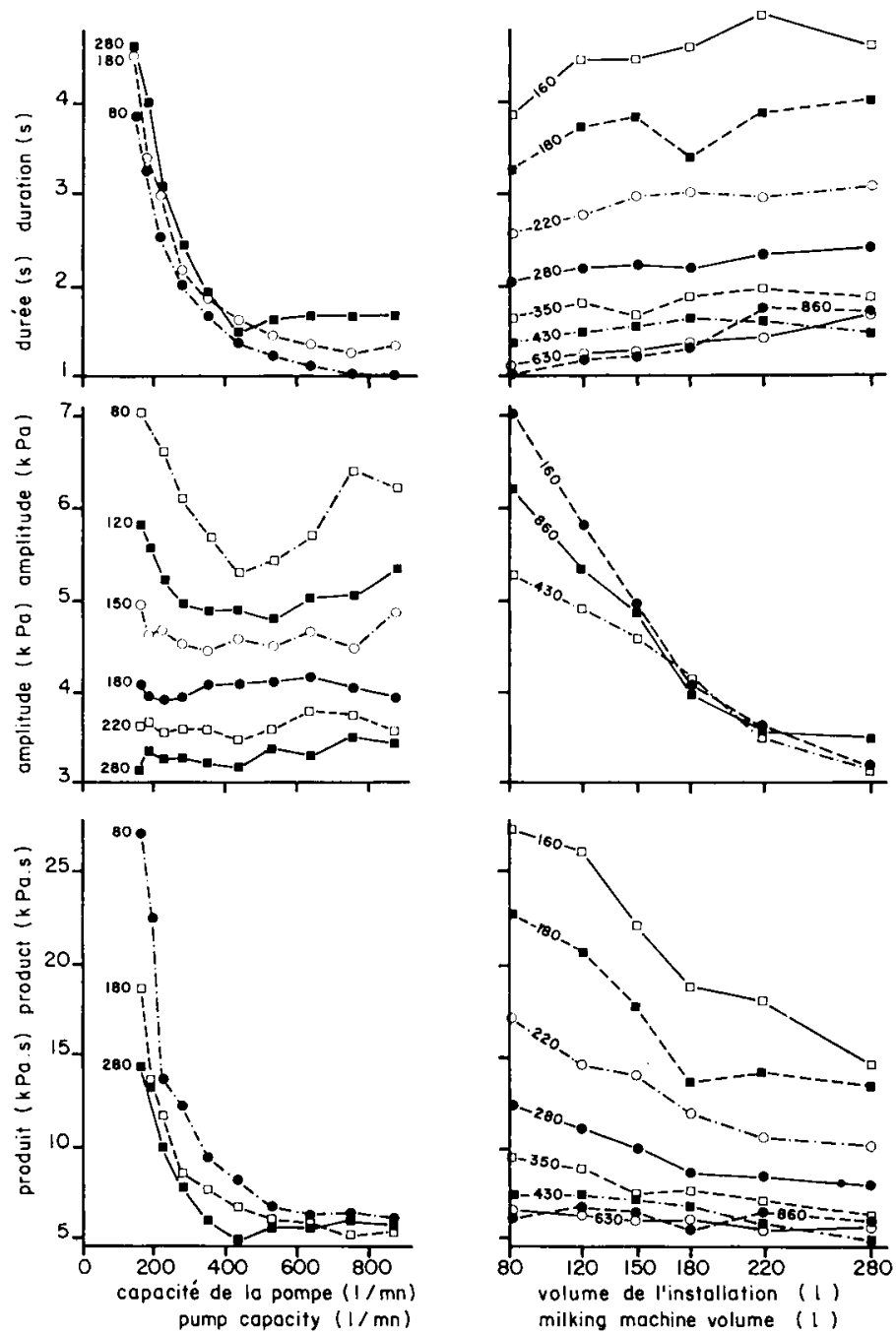

FIG. 3. - Influence de la capacité de la pompe à vide et du volume intérieur de l'installation sur la durée $\mathrm{d}$, l'amplitude a et le produit a $\times \mathrm{d}$ des perturbations du niveau de vide:

$\grave{a}$ gauche: variation de $d, a$ et $a \times d$ en fonction de la capacité de la pompe à vide pour des volumes intérieurs d'installation de $80,120,150,180,220$ et 2801 .

$\grave{a}$ droite : variation de $d, a$ et $a \times d$ en fonction du volume intérieur de l'installation pour des capacités de pompes à vide de r6o, I $80,220,280,35^{\circ}, 430,63^{\circ}$ et $8601 / \mathrm{mn}$.

Effect of vacuum pump capacity and plant internal volume on the duration $\mathrm{d}$, the amplitude a, and the product $\mathrm{a} \times \mathrm{d}$ of vacuum fiuctuations.

Left: variation of $d, a$ and $a \times d$ with the vacuum pump capacity for plant internal volumes of $80,120,150,180,220$ and $280 \mathrm{l}$.

Right: variation of $d$, $a$, and $a \times d$ with the plant internal volume for vacuum pump capacitics of $160,180,220,280,350,430,630$ and $8601 / \mathrm{mn}$. 


\section{C. - Amplitude des perturbations: a}

Quelle que soit la capacité de la pompe, l'accroissement du volume V se traduit par une diminution marquée de $a$ (fig. 3 et tabl. 2).

Pour un volume donné, l'effet d'une modification de la puissance de la pompe n'a qu'une faible incidence sur l'amplitude des ébranlements. Ainsi, pour les fortes valeurs de $V$, l'amplitude $a$ des perturbations croît avec $C$ (exemple : pour $V=2801$, l'amplitude $a$ passe de $3,15 \mathrm{kPa}$ pour $\mathrm{C}=160 \mathrm{Nl} / \mathrm{mn}$ à $3,49 \mathrm{kPa}$, pour $\mathrm{C}=$ $860 \mathrm{Nl} / \mathrm{mn}$ ) $(\mathrm{P}<0,05)$ (tabl. $\mathrm{I}$ ) tandis que pour les valeurs moyennes de $\mathrm{V}$ (exemple: $\mathrm{I} 8 \mathrm{o}$ l), $a$ est indépendant de la capacité de la pompe (tabl. I). Lorsque le volume $\mathrm{V}$ est faible (exemple : $80 \mathrm{l}$ ) il apparaît que l'accroissement de C entraîne tout d'abord une diminution $(P<O, O I)$ de $a$, puis une augmentation $(P<0$,or tabl. I ).

\section{D. - Produit de la durée par l'amplitude: $\mathrm{a} \times \mathrm{d}$}

La décroissance du produit $a \times d$ est d'autant plus faible que la capacité de la pompe et le volume de l'installation augmentent (fig. 3).

Ainsi pour un volume intérieur de 2801 , les produits $a \times d$ ne diffèrent pas

\section{TABLEAU 2}

Effet de l'accroissement du volume intérieur de l'installation (l) sur la durée, d, l'amplitude, a et le produit $\mathrm{a} \times \mathrm{d}$, des ébranlements pour des capacités de pompes de 160,350 et $860 \mathrm{l} / \mathrm{mn}$

Effect of increasing internal plant volume (1) on the duration d, the amplitude a, and the product $\mathrm{a} \times \mathrm{d}$, of the vacuum fuctuations for vacuum pumpcapacities of 160,350 and $860 \mathrm{l} / \mathrm{mn}$

\begin{tabular}{|c|c|c|c|c|c|c|c|c|c|c|}
\hline & & \multicolumn{9}{|c|}{$\begin{array}{c}\text { Capacité de pompe }(1 / \mathrm{mn}) \\
\text { Pump capacity }(l / m n)\end{array}$} \\
\hline & & \multicolumn{3}{|c|}{160} & \multicolumn{3}{|c|}{$35^{\circ}$} & \multicolumn{3}{|c|}{860} \\
\hline & & $d$ & $a$ & $a \times d$ & $d$ & $a$ & $a \times d$ & $d$ & $a$ & $a \times d$ \\
\hline$\Xi \Xi$ & 80 & 3,85 & 7,04 & 27,14 & $1,64^{a}$ & 5.72 & 9,41 & $0,9^{8}$ & 6,23 & 6,10 \\
\hline ثิ & 120 & 4,45 & 5,84 & 25,98 & $\mathrm{I}, 78$ & $4,9 \mathrm{I}$ & $8,0_{4}$ & $\mathrm{I}, 2 \mathrm{I}$ & $5.3^{6}$ & 5,52 \\
\hline 焉. & I 50 & 4,44 & 4,96 & 22,02 & $1,65^{a}$ & $4,4^{6}$ & 7,34 & $\mathrm{I}, 28$ & 4,89 & 7,09 \\
\hline$=\frac{8}{0}$ & I 80 & 4,57 & 4,08 & I 8,66 & I, 86 & 4,06 & 7,59 & 1,35 & 3.98 & 5,38 \\
\hline$\stackrel{\infty}{\Xi}$ & 220 & 4,92 & $3,6 \mathrm{r}$ & I 7,78 & $I, 95$ & 3.59 & 7,03 & $1,7 t$ & 3,60 & 6,33 \\
\hline$P \underset{\Sigma}{2}$ & 280 & 4,60 & 3,15 & 14,47 & I, 88 & 3,22 & 6,03 & 1,67 & 3,49 & 5,85 \\
\hline
\end{tabular}

Les valeurs réunies par un même trait vertical ou repérées par une même lettre ne diffèrent pas entre elles au niveau de probabilité de 5 p. Ioo. Du fait de l'hétérogénéité des variances, des tests " $t$ ont été effectués.

The values gathered by the same line or sharing a common letter are not significantly different $(\mathrm{P}>0,05)$.

Because of variance heterogeneity, the values were compared by means of the " $\mathrm{t}$ " test. 
significativement lorsqu'on accroît la capacité de la pompe de $35^{\circ} \mathrm{Nl} / \mathrm{mn}$ à $860 \mathrm{Nl} / \mathrm{mn}$ (tabl. I). De façon similaire, lorsque le volume intérieur d'une installation équipée d'une pompe de $860 \mathrm{l} / \mathrm{mn}$ passe de 801 à 2801 , la décroissance du produit $a \times d$ qui en résulte $(6,10 \mathrm{kPa} / \mathrm{s}$ à $5,85 \mathrm{kPa} / \mathrm{s})$ n'est pas significative (tabl. 2).

\section{Discussion}

La quantité d'air — Io $\mathrm{Nl}$ - admise dans une machine à traire en reliant au lactoduc un pot trayeur d'une contenance standard de 201 est voisine de la consommation nécessaire pour poser un faisceau-trayeur, estimée à $7,5 \mathrm{Nl}$ " si l'opérateur est habile " (Rossing, I970). Il cst en conséquence raisonnable de penser que les perturbations que nous avons enregistrées sont alors similaires à celles qui se produisent en pratique lors de l'usage normal d'une installation de traite. Aussi pour le contrôle à la ferme, une telle procédure nous apparaît mieux simuler le comportement réel du matériel que les méthodes basées sur la mesure du " temps de récupération "nécessaire pour compenser une chute de vide d'amplitude ou de durée déterminée. (Coguelin et Darracg, ig68; Mein, ig68; McDonald, i97I; Fluckiger et DE MARTini, I973).

En effet, alors qu'il est fréquemment admis que la stabilité du vide croît avec le volume intérieur de l'installation et la capacité de la pompe, on notera que dans nos conditions expérimentales :

- l'augmentation du volume intérieur se traduit, non pas par une diminution, mais par un accroissement faible, quoique parfois significatif, de la durée de l'ébranlement;

- l'amplitude des perturbations, bien que variant toujours en sens inverse du volume, semble dépendre assez peu de la capacité de la pompe.

Le choix de l'un de ces 2 critères utilisé seul comme représentatif de la stabilité du vide n'apparaît donc pas souhaitable.

En contrepartie, le produit de l'amplitude par la durée des fluctuations décroît lorsqu'on augmente la capacité $C$ de la pompe et le volume $V$ de l'installation. La décroissance est cependant d'autant plus forte que ces deux paramètres ont des valeurs faibles et à la limite, pour les pompes de plus fortes puissances le produit devient pratiquement indépendant de la capacité de la pompe et du volume intérieur de l'installation : il convient d'en tenir compte lors de l'interprétation des contrôles si l'on choisit d'utiliser ce critère pour caractériser la stabilité du vide. En outre, dans certaines installations, l'admission de l'air provoque plusieurs fluctuations successives du niveau de vide (fig. $2 b$ et $2 c$ ). Ce type d'instabilité, " rarement compris et auquel il est difficile de porter remède " (BLACKBURN, REETHOF, Shearer, I 966) est probablement dû aux forces en écoulement transitoire et pourrait résulter d'effets de résonance conditionnés par l'ensemble des composants de l'installation de traite. Or, ce phénomène est mis en évidence dans notre étude pour les pompes les plus puissantes, lorsque le régulateur fonctionne à la limite ou au-delà de sa capacité maximale. Ainsi, dans ce cas, additionner la durée et l'amplitude de plusieurs oscillations successives du vide pour déterminer la valeur du produit $a \times d$ conduirait notamment à une réduction apparente de l'influence de la capacité de la pompe sur la stabilité du vide (produit $a \times d$ ). Pour les tests à la ferme, une telle procédure n'apparaît donc pas souhaitable.

La rareté des études relatives à la stabilité du vide et la diversité des techniques utilisées rend difficile les comparaisons valables avec d'autres résultats. 
Toutefois, l'augmentation de la durée des perturbations avec le volume de l'installation est conforme aux conclusions de Whittlestone (I969) qui considère qu'un grand réservoir à vide est un inconvénient et écrit : "En théorie, les machines à traire les plus régulières ont un réservoir à vide très petit et la stabilité du vide dépend alors de la sensibilité du régulateur associé à une pompe de capacité suffisante ». De façon similaire, MuldownEy et O'CALLAGHAN (I970), recommandent le choix de valeurs $\frac{\mathrm{V}}{\mathrm{C}}$ aussi faibles que possible bien qu'ils notent que lorsque ce rapport est faible, l'amortissement du régulateur, difficile à réaliser, devient le principal facteur dont dépend la constance du niveau du vide. Enfin, selon PHILLIPS (I965), toute modification des pertes de charge entre la pompe (réservoir à vide) et le lactoduc influence la stabilité du vide. Or, dans nos conditions expérimentales les variations du volume intérieur de l'installation sont obtenues en agissant seulement sur la capacité du réservoir à vide. Il en résulte que nos résultats ne sont pas extrapolables aux cas où les changements du volume intérieur d'une installation seraient principalement dus à des modifications de longueur et de diamètre de canalisations.

\title{
Conclusion
}

Dans la gamme des valeurs étudiées, l'amélioration de stabilité du vide que l'on obtient en accroissant soit le volume intérieur, $\mathrm{V}$, soit la capacité de la pompe $\mathrm{C}$, est d'autant plus faible que $V$ et $C$ sont élevés. Il en résulte qu'au-delà d'un certain seuil l'amélioration devient nulle : augmenter le volume de roo 1 ou la capacité de la pompe de $400 \mathrm{Nl} / \mathrm{mn}$ n'entraîne, dans certains cas, aucun gain perceptible. Enfin, les caractéristiques du régulateur utilisé ainsi que la valeur des pertes de charge entre la pompe et le lactoduc nous semblent également susceptibles d'avoir une grande influence en ce qui concerne la stabilité du vide. Il conviendrait, par conséquent, d'approfondir l'étude de ces deux paramètres afin que l'éleveur puisse disposer de machines à traire plus stables; ceci au prix d'investissements qui demeurent toutefois en rapport avec l'amélioration à obterir.

Reçu pour publication en janvier 1976.

\section{Summary}

\author{
Variation in vacuum stability of a milking installation: \\ effect of pump capacity and internal plant volume
}

Milking vacuum stability was measured on an experimental milking machine with internaI machine volumes from $80 \mathrm{l}$ to 2801 and vacuum pump capacities from $\mathrm{I} 4 \mathrm{O} \mathrm{Nl} / \mathrm{mn}$ to $860 \mathrm{Nl} / \mathrm{mn}$.

From a bucket at atmospheric pressure (2ol), an amount of $10 \mathrm{~N} 1$ of air was rapidly introduced into the milkline while the installation was cperating (with liners stoppered) (fig. I). The spontaneous vacuum fluctuation which occurred, was recorded in the milkline and in the short milk tube on an oscillographic recorder by means of pressure transducers (fig. 2).

The results given in Figure 3 and Tables $I$ and 2 , show that:

- An increase in the vacuum pump capacity gave a significant increase in the duration of vacuum fluctuations $(P<0.0 I)$. A matked effect was observed for lower pump capacities, but pump capacity had a decreasing incidence for inner plant volume higher than $I_{5} \mathrm{O} I$.

- An increase of the internal volume gave a marked decrease in the amplitude of vacuum fluctuations $(P<0.0 I)$ and a little but significant increase $(P<O . O I)$ of the duration. 
- The vacuum stability represented by the product $p=$ amplitude $x$ duration was decreased for increasing pump capacity and plant volume.

However, for high pump capacities and plant volumes, this effect is relatively small. These limits could be partly due to the characteristics of the regulator and the loss in head between the vacuum pump and the milking units.

- Consequently, for mechanical testing of milking installations it seems possible to use the criterion $p=$ amplitude $\times$ duration for vacuum stability testing. However, with this procedure, it might be possible to obtain measurable differences between installations only for internal volumes and pump capacities of relatively small values.

\section{Références bibliographiques}

Blackburn J. F., Reethof G., Shearer J. L., 1966. Mécanismes et servomécanismes à fuide sous pression. 362-405, Dunod, Paris.

Coquelin M., Darrace J., ig68. Étude sur 1'emploi du test néozélandais de contrôle de la machine à traire, étude ronéotypée, I6 p. Inst. Tech. Elevage Bovin, Paris.

Fell L. R., 1963. Proc. Machine Milking School Conf., 108. Vict. Dept. Agric., Melbourne.

Fluckiger E., De Martini F., 1973. Le régulateur de vide de la machine à traire. Le Laitier Romand et Journal Suisse du Lait, 77, 591-592.

McDonald J. S., I97I. Relationship of milking machine design and function to udder disease. J. Amer. Vet. Med. Ass., 158, I84-19o.

Mein G. A., 1968. Proc. Symp. on machine milking, 271, National Inst. Res. in Dairying, Reading.

Muldowney C. J., O'Callaghan J. R., I97o, The interaction of components on the vacuum stability in milking machines. J. Agric. Engng. Res., 15, 331-346.

NyHAN J. F., I968. The effect of vacuum fluctuation on udder disease. Proc. Symp. Machine. Milking, 71-82, National Inst. Res. in Dairying, Reading.

Nyнan J. F., Cowhig M. J., I967. Inadequate milking machine vacuum reserve and mastitis. Vet Rec., 81, 1 22-124.

Phillips D. S. M., 1952. Air-flow meter for testing milking machines. N. Z. J. Agric., 85, $213-216$.

Philips D. S. M., 1965. Air flow in pipes in the milking machine. Publication $n^{\circ}$ I 85,7 p. From the proc. third Summer School and Conference on Machine Milking, Hobart.

RosSING W., I970. Air consumption of milking machine installations and the required capacity of the vacuum pump. Research Report 4, 9 p. Institute of Agricultural engineering and rationalization, Wageningen.

Rossing W., I973. Measurements of magnitude and duration of vacuum fluctuations caused by the regulator. Document ISO/TC $23 / S C I_{I} / W G, 2$ p., British Standards Institution, London.

Schmidt G. H., Switzer K. O., Guest R. W., Guthrie R. S., ig64. Effect of a teat-end vacuum fluctuation on milking rate and mastitis. J. Dairy Sci., 47, 761-765.

Stanley D. E., Kesler E. M., Borthree A., 1962. Effect of a fluctuating milking vacuum on certain measures of udder health. J. Dairy Sci., 45, I343-1347.

Whittlestone W. G., I969. Les principes de la traite mécanique. 57, Technipel S.A.R.L., Paris.

Woolford M. W., I974. Milking machine design: Factors affecting vacuum stability, (in abstract). Agricultural Research in the New Zealand. Annual report of research division, I972-1973, Ministry of the Agriculture and Fisheries, 79. 